\title{
A case report of simultaneous autoimmune and COVID-19 encephalitis
}

\author{
Fakhri Allahyari ${ }^{1} \cdot$ Ramin Hosseinzadeh $^{2} \cdot$ Javad Hosseini Nejad $^{1} \cdot$ Mohammad Heiat $^{3} \cdot$ Reza Ranjbar $^{4}$
}

Received: 14 November 2020 / Revised: 7 March 2021 / Accepted: 5 April 2021 / Published online: 26 April 2021

(c) Journal of NeuroVirology, Inc. 2021

\begin{abstract}
COVID-19 encephalitis is a rare condition usually presenting with altered mental status. Simultaneous presence of antiNMDAR antibody and SARS-CoV-2 virus in CSF is a very rare condition described in a few case reports so far. On the other hand, brain edema is an unusual presentation of anti-NMDAR encephalitis. Herein, we reported a case with simultaneous detection of anti-NMDAR antibody and SARS-CoV-2 virus in her cerebrospinal fluid (CSF) presenting with brain edema, altered mental status, seizures, and respiratory symptoms.
\end{abstract}

Keywords Autoimmune $\cdot$ COVID-19 $\cdot$ Encephalitis $\cdot$ Seizures

\section{Case report}

An 18-year-old girl with no medical history was admitted on June 22, 2020, to the emergency room of Ghaem hospital, Karaj, Iran, with generalized tonic-clonic seizures. She had a 3-week history of mood change as depression and anhedonia accompanied by lack of concentration, low-grade fever, dry coughs, and shortness of breath. Her respiratory symptoms were aggravated after the initiation of seizures. Despite the initiation of sodium valproate, she had a second episode of tonic-clonic seizures. At initial evaluations, she had a $38.7^{\circ} \mathrm{C}$ axillary fever, $100 / 60 \mathrm{mmHg}$ blood pressure, pulse rate of 106 , respiratory rate of 20 , oxygen saturation of $90 \%$, bilateral pulmonary crackles in lower lung zones, a confused state, minor meningismus, and neck stiffness.

Fakhri Allahyari and Ramin Hosseinzadeh contributed equally as first authors.

Reza Ranjbar

ranjbarre@gmail.com

1 Neuroscience Research Center, Baqiyatallah University of Medical Sciences, Tehran, Iran

2 Faculty of Medicine, Mashhad University of Medical Sciences, Mashhad, Iran

3 Baqiyatallah Research Center for Gastroenterology and Liver Diseases, Baqiyatallah University of Medical Sciences, Tehran, Iran

4 Molecular Biology Research Center, Systems Biology and Poisonings Institute, Baqiyatallah University of Medical Sciences, Tehran, Iran
She did not have any other neurological symptoms such as papillary edema, cranial nerve palsy, and focal neurological deficit on the neurological exam. Initial brain and lung CT scans are showed below (Fig. 1).

Meanwhile, her level of consciousness was deteriorated so she was undergone lumbar puncture and transmitted to ICU and started on broad spectrum antibiotics and acyclovir. CBC showed a WBC of 13,900 with neutrophilia (87\%) and lymphopenia(10\%). C-reactive protein was $2+$. According to these findings, she was started on Remdesivir, Lopinavir/Ritonavir, and interferon b1a (Resigen). A day after, CSF analysis came back as appearance: semi turbid, color: light pink, WBC: 27, RBC: 1997, lymphocytes: 93\%, PMN: 7\%, glucose: $55 \mathrm{mg} / \mathrm{dL}$, protein: $241 \mathrm{mg} / \mathrm{dL}$. HSV1\&2 DNA PCR also came back negative in CSF. No improvement in mental status and level of consciousness was seen in first 3 days but the seizures were controlled. At this time, an MRI was performed to have a closer look at the brain which came back normal and the brain edema was resolved (Fig. 2) so, she did not receive any specific treatments for the brain edema such as mannitol or hypertonic saline. Interestingly, her CSF PCR came back weakly positive for COVID-19. NMDAR antibody in CSF also came back positive in accordance with the result of indirect (transfected cells) immunofluorescence test (IIFT kit, EUROIMMUN Co., Lubeck, Germany). Therefore, she was started on IVIG ( $25 \mathrm{~g} / \mathrm{day})$. Her consciousness started to improve after $48 \mathrm{~h}$ of IVIG treatment. One week after admission, the blood and CSF cultures came back negative. Therefore, she was stopped on intravenous antibiotics 


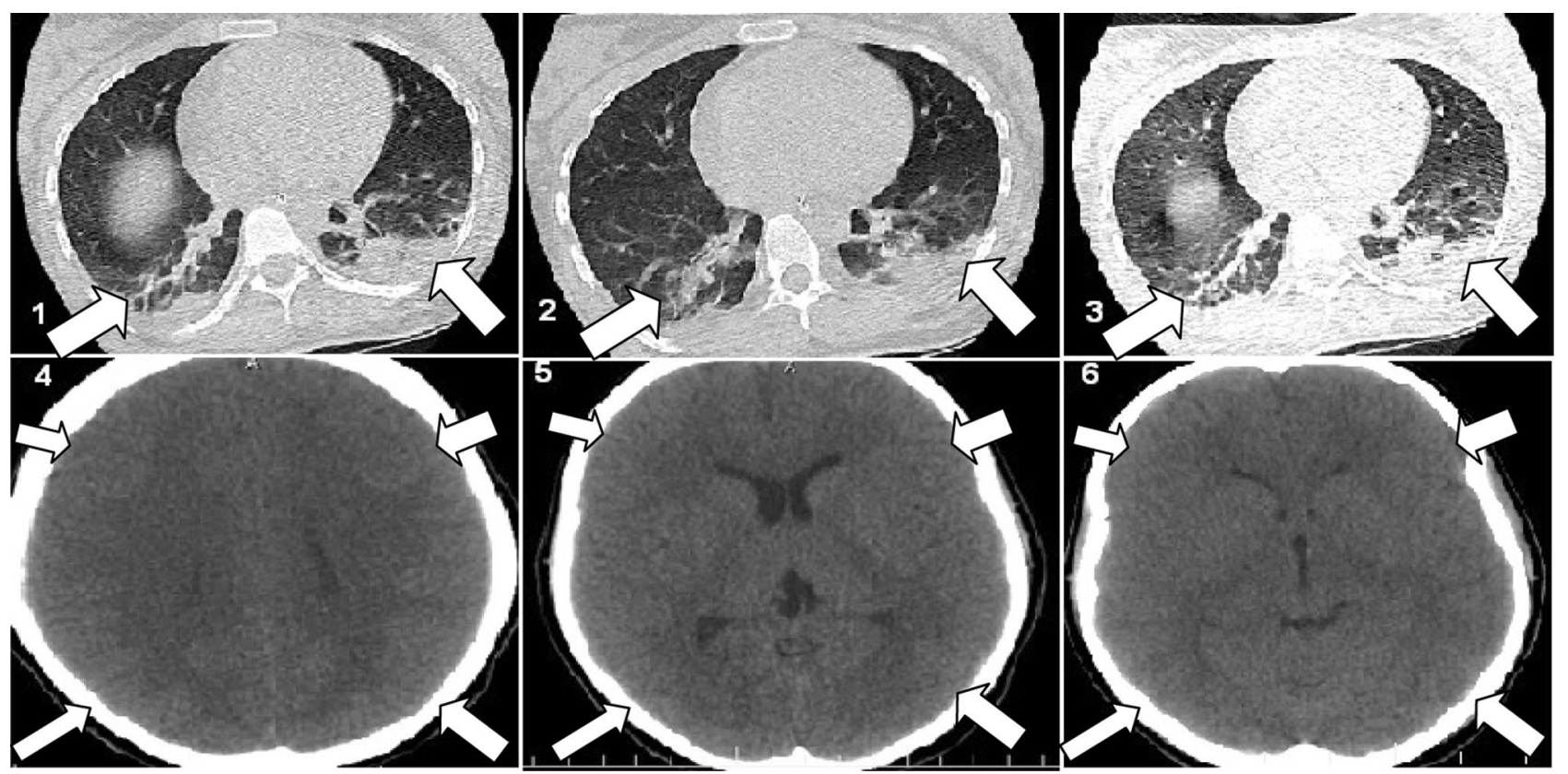

Fig. 1 Pictures 1-3 show peripheral ground-glass opacities and consolidations in lower lung zones. Pictures 4-5 show generalized brain edema

and started on methylprednisolone (2.5 g/5 days) and then tapered using oral corticosteroids. In the same time, her IgM for COVID-19 came back positive. After 2 weeks of admission, she was discharged from ICU and neurology service with normal consciousness. During hospitalization, her respiratory symptoms were mild and she did not show severe problems with oxygenation; therefore, there was no need to mechanical ventilation for the patient. After 2 months of hospitalization for completing COVID-19 treatments, she was discharged with full recovery.

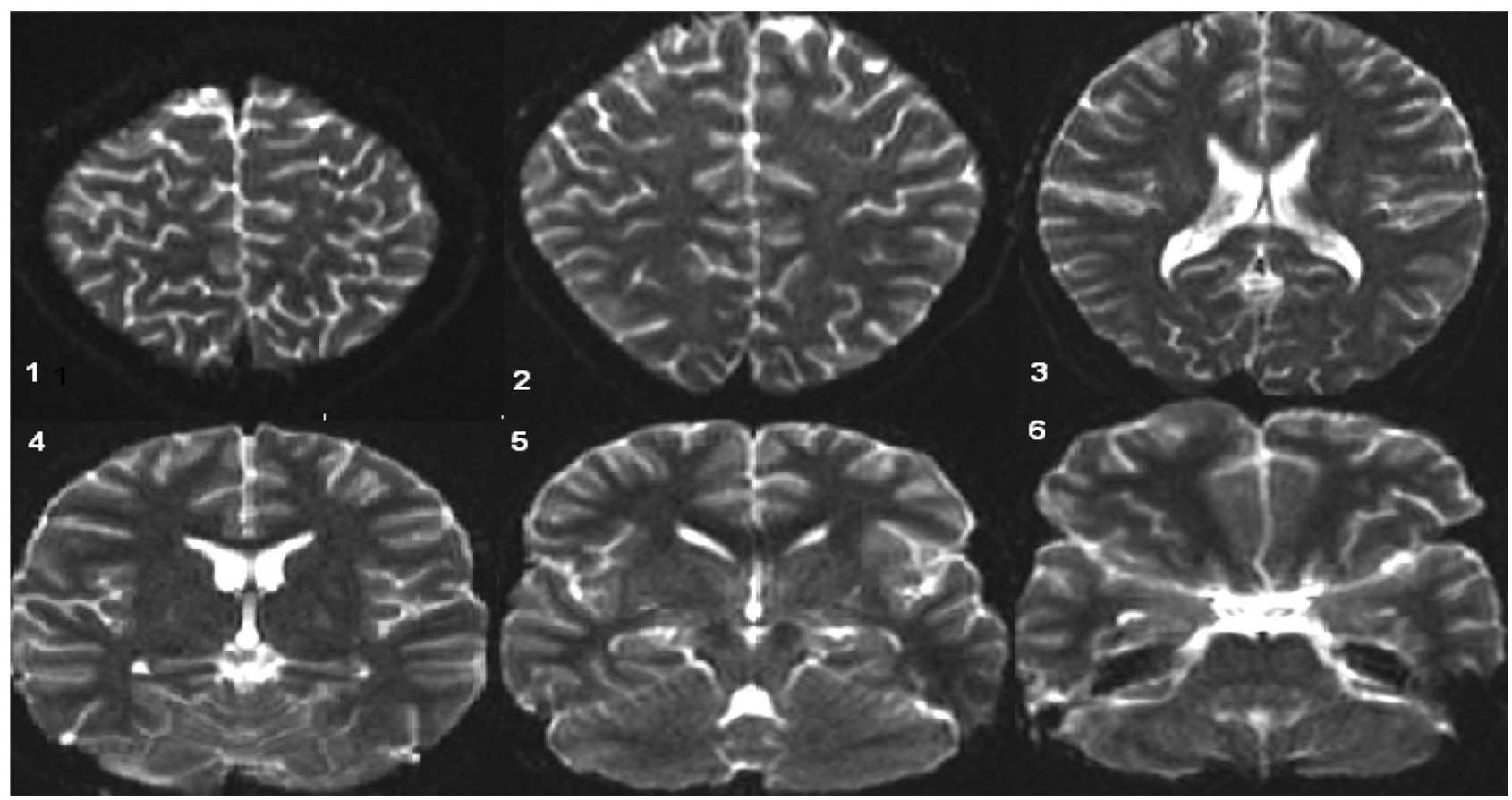

Fig. 2 Normal brain MRI. Pictures 1-6 show the brain edema has been resolved 


\section{Discussion}

Neurological symptoms of COVID-19 are caused by nervous system directly affected by the virus or immune-mediated para-infectious conditions (Romero-Sánchez et al. 2020; Helms et al. 2020; Moriguchi et al. 2020). Anti-NMDAR encephalitis has been reported in few cases of COVID-19 before (Panariello et al. 2020; Monti et al. 2020; Burr et al. 2020). On the other hand, brain edema which is an unusual finding in anti-NMDAR encephalitis has also been rarely reported in COVID-19 patients (Coolen et al. 2020; Kadono et al. 2020). In this case, we had simultaneous presence of SARS-CoV-2 RNA and NMDAR antibody in CSF accompanied by generalized brain edema which is rare, and to our knowledge, it has never been addressed so far. Although many new insights have been provided in regard to the pathogenesis of COVID-19, precise interactions between virus-host cells remain unclear. Indeed, the integration of new emerging sciences will help us to fight COVID-19 disease and related complications more efficiently in the future. (Mohammadpour et al. 2021; Mirzaei et al. 2020a; Heiat et al. 2021; Mirzaie et al. 2020b).

Acknowledgements We would like to thank the Clinical Research Development Unit of Baqiyatallah University of Medical Science for their kindly cooperation.

\section{Declarations}

Conflict of interest The authors declare no competing interests.

\section{References}

Burr T, Barton C, Doll E, Lakhotia A, Sweeney M (2020) NMDAreceptor encephalitis associated with COVID-19 infection in a toddler. Pediatric Neurol
Coolen T, Lolli V, Sadeghi N, Rovai A, Trotta N, Taccone FS et al (2020) Early postmortem brain MRI findings in COVID-19 nonsurvivors. Neurology 95(14):e2016-e2027

Heiat M, Hashemi-Aghdam MR, Heiat F, Rastegar Shariat Panahi M, Aghamollaei H, Moosazadeh Moghaddam M, Thozhukat Sathyapalan, Reza Ranjbar, Sahebkar A (2021) Integrative role of traditional and modern technologies to combat COVID-19. Expert Review of Anti-infective Therapy 19 (1):23-33

Helms J, Kremer SŠ, Merdji H, Clere-Jehl Rl, Schenck M, Kummerlen $\mathrm{C}$ et al (2020) Neurologic features in severe SARS-CoV-2 infection. New England J Med

Kadono Y, Nakamura Y, Ogawa Y, Yamamoto S, Kajikawa R, Nakajima $Y$ et al (2020) A case of COVID-19 infection presenting with a seizure following severe brain edema. Seizure 80:53

Mirzaie A, Halaji M, Dehkordi FS, Ranjbar R, Noorbazargan H (2020a) A narrative literature review on traditional medicine options for treatment of corona virus disease 2019 (COVID-19). Complementary Therapies in Clinical Practice 40:101214

Mirzaei R, Karampoor S, Sholeh M, Moradi P, Ranjbar R, Ghasemi F (2020b) A contemporary review on pathogenesis and immunity of COVID-19 infection. Mol Biol Rep 47 (7):5365-5376

Mohammadpour S, Torshizi Esfahani A, Halaji M, Lak M, Ranjbar R (2021) An updated review of the association of host genetic factors with susceptibility and resistance to COVID-19 J Cell Physiol 236:49-54

Monti G, Giovannini G, Marudi A, Bedin R, Melegari A, Simone AM et al (2020) Anti-NMDA receptor encephalitis presenting as new onset refractory status epilepticus in COVID-19. Seizure 81:18

Moriguchi T, Harii N, Goto J, Harada D, Sugawara H, Takamino J, Nakao A (2020) A first case of meningitis/encephalitis associated with SARS-Coronavirus-2. International Journal of Infectious Diseases

Panariello A, Bassetti R, Radice A, Rossotti R, Puoti M, Corradin M et al (2020) Anti-NMDA receptor encephalitis in a psychiatric Covid-19 patient: a case report. Brain, behavior, and immunity

Romero-Sánchez CM, Díaz-Maroto I, Fernández-Díaz E, SánchezLarsen Á, Layos-Romero A, García-García J et al (2020) Neurological manifestations in hospitalized patients with COVID-19: a retrospective. observational study from a Spanish population. The ALBACOVID Registry

Publisher's Note Springer Nature remains neutral with regard to jurisdictional claims in published maps and institutional affiliations. 